\title{
ANTIFUNGAL PROPHYLAXIS WITH POSACONAZOLE IN IMMUNOCOMPROMISED CHILDREN UNDER 13 YEARS OF AGE IN A REFERENCE HOSPITAL IN LIMA, PERU
}

\author{
Julio Maquera-Afaray ${ }^{1}$, Medalit Luna-Vilchez ${ }^{1}$, Blanca Salazar-Mesones ${ }^{1}$, Diana \\ Portillo-Alvarez ${ }^{1}$, Luis Uribe- Ramirez ${ }^{1}$, Graciela Taipe-Sedano ${ }^{1}$, Carlos Santillán-Salas ${ }^{1}$, \\ and José López ${ }^{1}$ \\ ${ }^{1}$ Instituto Nacional de Salud del Niño San Borja
}

September 16, 2020

\begin{abstract}
Background: Prophylaxis with posaconazole (PP) has shown to be effective in the prevention of invasive fungal infections in immunocompromised adult patients. However, evaluation of its effectiveness and safety in children is limited. The aim of the study was to describe the use of posaconazole as antifungal prophylaxis in children. Methods: We reviewed the medical records of immunocompromised patients under 13 years of age with hematological diseases and post hematopoietic stem cell transplantation (HSCT) who received antifungal PP at the Instituto Nacional de Salud del Niño San Borja (Perú) from January 2014 to December 2018. Results: Fifty-six antifungal prophylaxis events were identified in 47 patients with a median age of 7.5 years (interquartile range [IQR] $4-10), 51.6 \%(\mathrm{n}=24)$ of whom were female. The main underlying medical conditions were aplastic anemia $(\mathrm{n}=19,33.9 \%)$, acute lymphoblastic leukemia $(\mathrm{n}=18,32.1 \%)$, acute myeloid leukemia $(\mathrm{n}=14,25.0 \%)$, and $34.1 \%$ had undergone HSCT. The median dose of posaconazole was $13.62 \mathrm{mg} / \mathrm{kg} / \mathrm{day}$ (IQR: 12.0-16.8), and the median duration of PP was 24 days (IQR: 16-82). Gastrointestinal symptoms included abdominal pain (17.9\%), nausea (16.1\%), diarrhea (7.1\%) and vomiting $(3.6 \%)$. Elevated alanine aminotransferase and aspartate aminotransferase levels were observed in 9/35 patients $(25.7 \%)$ and 10/51 (19.6\%) patients, respectively. Five cases of breakthrough fungal infection were identified (8.9\%). Conclusions: Breakthrough fungal infection, increased transaminase levels and gastrointestinal symptoms were observed during PP in children under 13 years of age.
\end{abstract}

\section{INTRODUCTION}

The use of prophylactic antifungals has dramatically decreased the risk of the development of invasive fungal infections (IFIs) in cancer patients and patients undergoing hematopoietic stem cell transplantation (HSCT) being a major cause of morbidity and mortality $(1,2)$.

Posaconazole is a triazole-extended spectrum used primarily for the prophylaxis and treatment of IFIs refractory to other antifungal therapies, due to its extensive coverage of yeast and filamentous fungi, and has shown to be effective in adolescents and adults (2-4). This drug is only approved for use in adults in Europe, and the oral suspension is approved by the Food and Drug Administration (FDA) only in individuals over 13 years of age $(3,4)$. The recommendation for the use of posaconazole in children under 13 years of age is limited to individual cases as an "off- label" prescription (5). Therefore, the use of posaconazole in prophylaxis (PP) in children has not been fully evaluated, and this is important since the underlying risk of drug toxicity and poor adherence to the drug could impact the failure of prophylaxis, increasing the risk of breakthrough fungal infection (3-5).Nonetheless, while the efficacy, safety, and pharmacokinetic characteristics of posaconazole as a prophylactic alternative have been evaluated in high-risk pediatric patients $(6,7)$, it is only recommended 
for use in patients over the age of 13 years, unlike other antifungal drugs such as fluconazole, itraconazole, and voriconazole $(6,7)$.

The aim of this study was to describe our experience with PP in children under 13 years of age with hematological diseases who had undergone HSCT at the Instituto Nacional de Salud del Niño San Borja (INSN-SB) in Lima, Peru.

\section{METHODS}

\section{Setting:}

The Instituto Nacional de Salud del Niño San Borja is a pediatric referral hospital located in Lima, Peru which provides care for children and adolescents with hematologic diseases, and also patients undergoing HSCT. The Department of Clinical Hematology has 24 beds and a transplantation unit with 20 beds.

\section{Study design:}

Retrospective review of the medical charts of patients.

Study population and selection criteria:

From January 2014 to December 2018, all patients treated in our institution under 13 years of age with hematological diseases who underwent HSCT and received antifungal PP were included in the study. Patients with incomplete data for the variables analyzed in the medical record were excluded.

\section{Study variables:}

Demographic variables such as age, sex, underlying oncologic or hematological disease, reception of HSCT, and development of graft-versus-host disease were recorded. PP was defined as antifungal prophylaxis using posaconazole (in oral suspension) for more than seven consecutive days in a patient without symptoms suggestive of invasive fungal infection.

Variables associated with toxicity and the effectiveness of PP were also registered, including the prophylactic dose used, concomitant use of proton pump inhibitors, presence of mucositis, clinical manifestations (while using PP), liver enzyme values - alanine-aminotransferase (ALT) and aspartate aminotransferase (AST)and the history and development of new fungal infection during antifungal prophylaxis (breakthrough fungal infection). Liver function test values were divided into 3 periods: before (AST and ALT tests obtained 2 weeks before the initiation of PP); during (maximum AST and ALT values during PP); and after PP (AST and ALT tests performed up to 2 weeks after discontinuing PP). To determine and classify invasive fungal infections as possible, probable, and proven, the diagnostic criteria of the European Organization for Research and Treatment of Cancer and Mycoses Study Group of the National Institute of Allergy and Infectious Diseases (EORTC/MSG) were used (8). No data regarding posaconazole plasma levels were collected because the test was not available at the institution during the data collection period.

\section{Data analysis:}

Descriptive statistics of variables were expressed as medians and interquartile range (IQR), according to the analysis of the distribution of data, while qualitative variables were summarized using frequency and percentages. Second, ALT and AST values were analyzed in three periods: before, during, and after the suspension of PP to establish differences in these periods. For the overall analysis of the three periods the Kruskal Wallis and Mann Whitney U tests were used to determine differences between 2 periods (before vs. during, during vs. after, and before vs. after). The Stata(r) v16 statistical software (Stata Corporation, College Station, Texas, USA) was used for the analyses. A value of $\mathrm{p}<0.05$ was considered statistically significant.

\section{Ethical aspects :}

The study protocol was approved by the Institutional Review Board of the INSN-SB, under the institutional code PI-210-2018. 


\section{RESULTS}

Fifty-six PP events were identified in 47 patients. The mean age of the patients was 7.5 years (IQR 4-10), and $24(51.6 \%)$ were female. The underlying medical conditions were aplastic anemia $(\mathrm{n}=19,33.93 \%)$, acute lymphoid leukemia $(\mathrm{n}=18,32.14 \%)$, acute myeloid leukemia $(\mathrm{n}=14,25.0 \%)$, Fanconi anemia $(\mathrm{n}=2,3.57 \%)$, thalassemia $(\mathrm{n}=1,1.79 \%)$, primary immunodeficiency $(\mathrm{n}=1,1.79 \%)$, and myelodysplastic syndrome $(\mathrm{n}=1$, $1.79 \%$ ). In addition, $34.1 \%$ underwent HSCT (haploid: $21.3 \%$; allogenic: $12.8 \%$ ) and of these, 6 (10.71\%) developed GVHD.

The median dose of posaconazole used was $13.6 \mathrm{mg} / \mathrm{kg} /$ day (IQR 12 - 16.8). The median duration of PP was 24 days (IQR 16- 82), with. 33.9\% concomitantly using proton pump inhibitors and $7.14 \%$ had mucositis. The median absolute neutrophil count before the beginning PP was 600 cells/mL (IQR: 150- 1980). The clinical and laboratory findings during the use of PP are shown in Table 1.

There were significant difference between the median ALT values before (33.5, IQR: 13-61.5), during (70, IQR: 43-133) and after discontinuation (39, IQR: 25.5-79.5) of PP ( $\mathrm{p}<0.001)$, and when comparing the values during prophylaxis with the values before $(\mathrm{p}<0.001)$ and after PP suspension $(\mathrm{p}<0.001)$. No significant differences were found between the median ALT levels before and after stopping PP $(\mathrm{p}=0.205)$. Significant differences were observed between the median AST levels before (30, IQ: 21-43), during (59, IQ: 35-103.5), and after the discontinuation (31.5, IQ: 22-46) of PP $(\mathrm{p}<0.001)$. Likewise, there were significant differences between the values during prophylaxis compared with the values before $(\mathrm{p}<0.001)$ and after the suspension of PP $(\mathrm{p}<0.001)$. There were no significant differences between the median ALT levels before and after stopping PP $(\mathrm{p}=0.552)$ (Figure 1$)$.

We identified five breakthrough fungal infections, four of which $(4 / 5)$ corresponded to invasive aspergillosis (2 probable and 2 possible), and one (1/5) invasive candidiasis. Of the total patients, 15 (31.91\%) had a prior history of IFI (11 invasive aspergillosis and 4 invasive candidiasis). Table 2 shows the characteristics of the cases presenting breakthrough fungal infections.

\section{DISCUSSION}

In the present study, transaminase values increased 2- to 3-fold during PP use, and five patients (8.9\%) presented breakthrough fungal infections.

Oral posaconazole is an alternative 'off-label' medication for patients under 13 years of age. However, there are concerns related to the bioavailability of the drug, which may be variable, making it difficult to achieve optimal plasma levels of posaconazole in pediatric patients, even with an adequate dose regimen (9). Our patients received dosing schemes similar to those reported in previous studies $(12 \mathrm{mg} / \mathrm{kg} /$ day) (10.11), with the variations observed in our institution being due to a non-standardized prescription regimen during the study period. On the other hand, a previous study demonstrated that doses less than $12 \mathrm{mg} / \mathrm{kg} / \mathrm{day}$ could be related to suboptimal posaconazole plasma levels (values less than $0.7 \mu \mathrm{g} / \mathrm{mL}$ ) (12). In contrast, another study, which administered oral posaconazole to pediatric patients, found that only $31 \%$ of patients aged 2 to 6 years achieved optimal average steady-state posaconazole concentrations (calculated as the area under the curve (AUC)/dose interval in 24 hours) between 500 and $2500 \mathrm{ng} / \mathrm{mL}$, using doses of $12 \mathrm{mg} / \mathrm{Kg} /$ day, with a dosing interval of 2 times per day (7).

In this regard, some authors suggest an increase in the frequency of the dose interval to achieve optimal plasma levels due to the saturable bioavailability of posaconazole (13). An initial dose of PP of $200 \mathrm{mg} 3$ times a day (oral suspension) is recommended in pediatric populations between 6 months to 6 years of age, while in children between 7 and 12 years, $300 \mathrm{mg} 3$ times a day is recommended in the case of oral suspension (for children who can not take pills), or $200 \mathrm{mg}$ three times a day, in the case of pills, increasing the dose if the therapeutic drug monitoring (TDM) is less than $0.7 \mathrm{mg} / \mathrm{L}(13)$.

Other factors can affect the absorption of posaconazole, such as the concomitant use of proton pump inhibitors and the presence of mucositis, which limit the achievement of optimal prophylactic and therapeutic 
levels $(14,15)$. One study reported that the use of proton pump inhibitors was associated with a $42 \%$ reduction in posaconazole bioavailability in children younger than 13 years (13). In our study, $33.9 \%$ used proton pump inhibitors and $7.1 \%$ developed mucositis during PP use. The bioavailability of posaconazole was not analyzed in this study, therefore it is not possible to evaluate the effect of the use of proton pump inhibitors and the presence of mucositis on this variable.

Concerning possible adverse events due to the use of posaconazole, several studies have described clinical findings similar to ours, corresponding mainly to gastrointestinal manifestations such as abdominal pain and nausea $(10,11,16)$. On the other hand, the 2- to 3 -fold increase in ALT and AST values observed in our study has also been described in other studies carried out in populations of children who received posaconazole $(16,17)$. In addition, comparative studies of posaconazole and other azoles employed for antifungal prophylaxis in pediatric patients described the development of adverse events such as hepatotoxicity (10), reporting a significant increase in ALT and AST values from the serum value at the beginning and the maximum values during the use of different groups of azoles, including posaconazole (11).

In contrast, other studies observed only one case of breakthrough fungal infection by Aspergillus fumigatus $(3.0 \%)$, in patients under 18 years of age during PP use (17), with other series in children reporting no case of breakthrough fungal infection during PP $(6,16)$. In a study comparing itraconazole, voriconazole, and posaconazole used as oral antifungal prophylaxis in pediatric patients undergoing HSCT, no case of breakthrough fungal infection was found in the posaconazole group, while in the itraconazole and voriconazole groups there were $4 \%$ and $6 \%$ of possible cases of IFI, respectively (11). We found a higher number of breakthrough fungal infection cases ( 5 cases; $8.9 \%$ ) compared to the previously described reports. It should be noted that three of these cases concomitantly used proton pump inhibitors, two did not receive the drug along with high-fat meals, and one had mucositis. This is important,since it has previously been described that these variables can decrease the bioavailability of the drug and thereby favor the development of breakthrough fungal infection (15).

In patients younger than 13 years, the main prophylactic antifungal agents (with activity against filamentous fungi) recommended are itraconazole and voriconazole with the use of TDM, in both cases $(3,18)$. Posaconazole is an ' off-label' alternative in this group of patients, and it also requires TDM to ensure adherence and adequate exposure to the drug, especially when used in oral suspension and in children under 13 years of age (19). However, liver toxicity and breakthrough fungal infection episodes developed in some patients during the use of posaconazole in our study. To date, in Peru, no public health center performs TDM for antifungals, and in most health centers the oral suspension presentation of voriconazole is not available as a recommended prophylactic alternative in children older than 2 years (18).

The clinical and laboratory findings in our study may not only be due to the use of posaconazole, but also to the use of another medication or interaction with other drugs used in this group of patients. Furthermore, TDM of posaconazole was not performed and therefore a relationship between the adverse events observed (clinical, laboratory, and breakthrough fungal infection) and the plasma levels of the drug (supra-therapeutic or infra-therapeutic) could not be determined.

In conclusion, patients less than 13 years of age receiving PP showed an increase in transaminase values, and the development of breakthrough fungal infections, demonstrating the need for TDM during this type of prophylactic treatment.

\section{CONFLICTS OF INTEREST:}

The authors do not declare any conflict of interest in the present study.

\section{ACKNOWLEDGMENTS:}

The authors thank Dr. Carlos Ramírez Vallejos for his collaboration in the study.

\section{REFERENCES}


1. Castagnola E, Mesini A. Antifungal Prophylaxis in Children Receiving Antineoplastic Chemotherapy. Curr Fungal Infect Rep. 2018;12(2):78-85.

2. Lehrnbecher T. Antifungal prophylaxis in pediatric patients undergoing therapy for cancer: drugs and dosing. Curr Opin Infect Dis. 2015;28(6):523-31.

3. Groll AH, Castagnola E, Cesaro S, et al. Fourth European Conference on Infections in Leukaemia (ECIL4): guidelines for diagnosis, prevention, and treatment of invasive fungal diseases in paediatric patients with cancer or allogeneic haemopoietic stem-cell transplantation. Lancet Oncol. 2014;15(8):e327-340.

4. Ullmann AJ, Schmidt-Hieber M, Bertz H, et al. Infectious diseases in allogeneic haematopoietic stem cell transplantation: prevention and prophylaxis strategy guidelines 2016. Ann Hematol. 2016;95(9):1435-55.

5. Vicenzi EB, Cesaro S. Posaconazole in immunocompromised pediatric patients. Expert Rev Anti Infect Ther. 2018;16(7):543-53.

6. Döring M, Cabanillas Stanchi KM, Queudeville M, et al. Efficacy, safety and feasibility of antifungal prophylaxis with posaconazole tablet in paediatric patients after haematopoietic stem cell transplantation. J Cancer Res Clin Oncol. 2017;143(7):1281-92.

7. Arrieta AC, Sung L, Bradley JS, et al. A non-randomized trial to assess the safety, tolerability, and pharmacokinetics of posaconazole oral suspension in immunocompromised children with neutropenia. PloS One. 2019;14(3):e0212837.

8. De Pauw B, Walsh TJ, Donnelly JP, et al. Revised definitions of invasive fungal disease from the European Organization for Research and Treatment of Cancer/Invasive Fungal Infections Cooperative Group and the National Institute of Allergy and Infectious Diseases Mycoses Study Group (EORTC/MSG) Consensus Group. Clin Infect Dis Off Publ Infect Dis Soc Am. 2008;46(12):1813-21.

9. Gwee A, Cranswick N, Curtis N. Posaconazole: promising but problematic in practice in pediatric patients. Pediatr Infect Dis J. 2015;34(6):604-6.

10. Döring M, Eikemeier M, Cabanillas Stanchi KM, et al. Antifungal prophylaxis with posaconazole vs. fluconazole or itraconazole in pediatric patients with neutropenia. Eur J Clin Microbiol Infect Dis. 2015;34(6):1189-200.

11. Döring M, Blume O, Haufe S, et al. Comparison of itraconazole, voriconazole, and posaconazole as oral antifungal prophylaxis in pediatric patients following allogeneic hematopoietic stem cell transplantation. Eur J Clin Microbiol Infect Dis. 2014;33(4):629-38.

12. Valenzuela R, García P, Barraza M, et al. [Pharmacokinetics of posaconazol in the prophylaxis and treatment of invasive fungal infection in immunocompromised children in a pediatric hospital]. Rev Chil Infectologia Organo Of Soc Chil Infectologia. 2018;35(1):15-21.

13. Boonsathorn S, Cheng I, Kloprogge F, et al. Clinical Pharmacokinetics and Dose Recommendations for Posaconazole in Infants and Children. Clin Pharmacokinet. 2019;58(1):53-61.

14. Dolton MJ, Brüggemann RJM, Burger DM, et al. Understanding variability in posaconazole exposure using an integrated population pharmacokinetic analysis. Antimicrob Agents Chemother. 2014;58(11):687985 .

15. Sienkiewicz-Oleszkiewicz B, Urbańczyk K, Stachowiak M, et al. Factors Influencing the Safety and Efficiency of Antifungal Prophylaxis with Posaconazole in Children with Hematological Diseases: From Genetics to Polypharmacotherapy. Indian J Hematol Blood Transfus. 2019;35(4):699-706.

16. Döring M, Müller C, Johann P-D, et al. Analysis of posaconazole as oral antifungal prophylaxis in pediatric patients under 12 years of age following allogeneic stem cell transplantation. BMC Infect Dis. 2012;12:263. 
17. Döring M, Cabanillas Stanchi KM, Klinker H, et al. Posaconazole plasma concentrations in pediatric patients receiving antifungal prophylaxis during neutropenia. Med Mycol. 2017;55(4):375-84.

18. Ramos JT, Romero CA, Belda S, et al. Clinical practice update of antifungal prophylaxis in immunocompromised children. Rev Esp Quimioter. 2019;32(5):410-25.

19. Jancel T, Shaw PA, Hallahan CW, et al. Therapeutic drug monitoring of posaconazole oral suspension in paediatric patients younger than 13 years of age: a retrospective analysis and literature review. J Clin Pharm Ther. 2017;42(1):75-9.

\section{Hosted file}

Table 1..docx available at https://authorea.com/users/359549/articles/481457-antifungalprophylaxis-with-posaconazole-in-immunocompromised-children-under-13-years-of-age-in-areference-hospital-in-lima-peru

\section{Hosted file}

Table 2..docx available at https://authorea.com/users/359549/articles/481457-antifungalprophylaxis-with-posaconazole-in-immunocompromised-children-under-13-years-of-age-in-areference-hospital-in-lima-peru

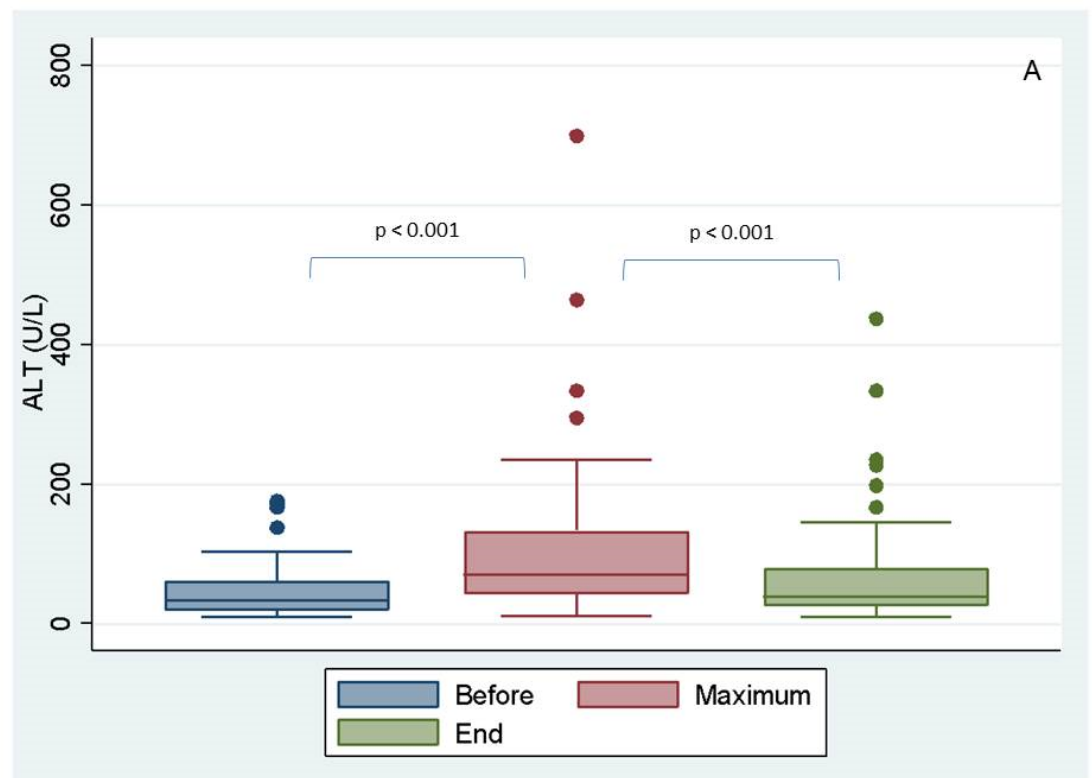




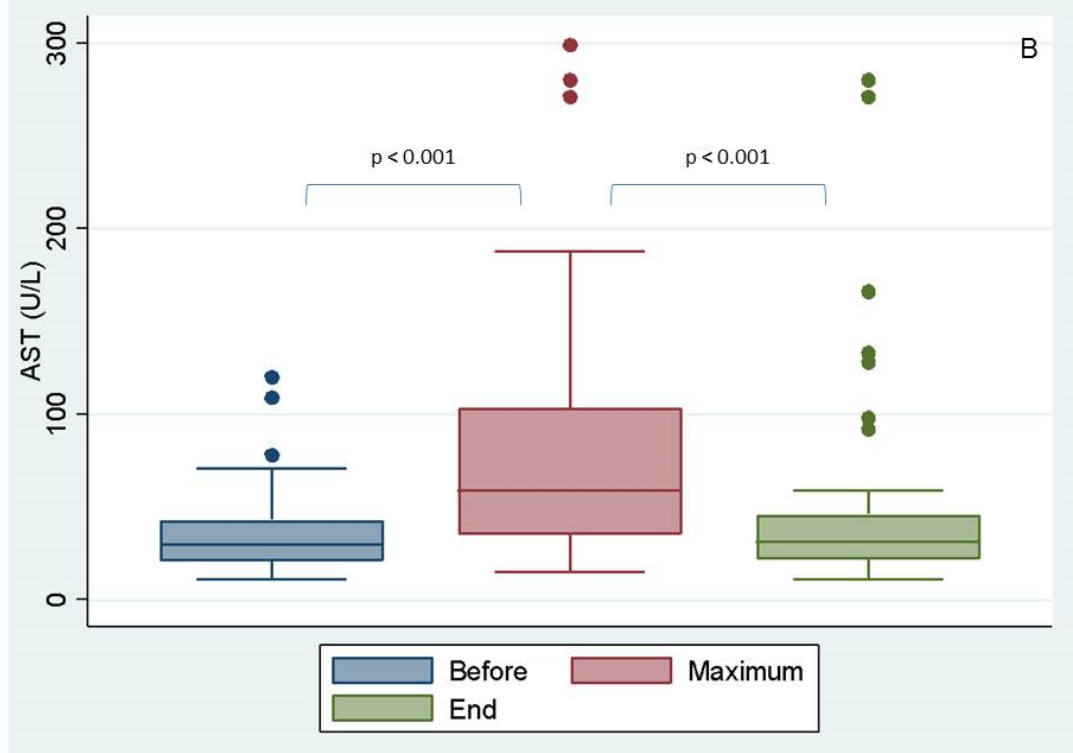

